\title{
Optimization of process parameters influencing the sustainable construction of iron oxide nanoparticles by a novel tropical wetlands Streptomyces spp.
}

\begin{abstract}
A Streptomyces strain isolated from the soil sediments of tropical freshwater wetlands in Malaysia demonstrated promising attributes to be developed into a versatile microbial nanofactory for the sustainable synthesis of ferric oxide nanoparticles (IONP). Process parameters such as temperature, ferric salt precursor concentration, cell free extract (CFE) concentration and biomass harvesting times are serious players in the extracellular generation of metallic nanoparticles. A statistical approach using One-Variable-At-A-Time (OVAT) Analysis followed by Response Surface Methodology (RSM) was employed towards the optimization of the microbial bioprocess and modulation of nanoparticle size dimensions. OVAT revealed that IONP production increased with increasing temperature, precursor concentration, harvesting time and CFE concentration with highest yield at $65^{\circ} \mathrm{C}, 2 \mathrm{mM}$ precursor concentration, $68 \mathrm{~h}$ harvesting time and 100\% CFE concentration. A detailed statistical analysis using RSM (RSM) showed significantly strong negative interactive effects between temperature and CFE concentration $(p=0.0037)$, temperature and precursor concentration $(p=0.0093)$ and mild interactive effects between CFE and precursor concentration $(\mathrm{p}=0.0301)$. Taking into account the interactive influence of these variables, numerical analysis using RSM proposed that for optimal generation of microbial mediated IONP, a CFE concentration of $55.58 \%$, temperature of $55.75^{\circ} \mathrm{C}$ and precursor concentration of $2.46 \mathrm{mM} \mathrm{FeCl3.6H2O}$ would be required.
\end{abstract}

Keyword: Melanin induced; Bionanoparticles; Optimization; Size; Polydispersity; Temperature; Biomass; Precursor concentration; Reaction kinetics 